\title{
Arquitectura y casos de uso de nubes híbridas en entornos regulados
}

\author{
Architecture and use cases of hybrid clouds in regulated environments
}

Recibido: noviembre 22 de 2021 | Revisado: noviembre 26 de 2021 | Aceptado: diciembre 06 de 2021

\author{
Richard Miguel San Martín ${ }^{\mathrm{I}}$ \\ José Antonio Wu Chong ${ }^{\mathrm{I}}$
}

1 Facultad de Ingeniería y Arquitectura. Universidad de San Martín de Porres (USMP). Lima, Perú

Autor para correspondencia

E-mail: richard_miguel@usmp.pe

\begin{abstract}
Resumen
El objetivo de este artículo es el de proponer un método de arquitectura que incorpore elementos tecnológicos de uso común en la implementación de nubes híbridas y que permita cerrar la brecha en aspectos de diseño en soluciones para empresas que se desempeñan en industrias con regulaciones. En la actualidad, el uso de la nube pública es el servicio con mayor crecimiento de mercado, orientando el uso de servicios a demanda, conocidos como XasS (All As a Service). En este escenario donde los datos se transfieren a nubes públicas, se incrementa la preocupación de los usuarios en cómo hacer uso seguro de la tecnología disponible para Cloud cumpliendo con regulaciones propias de negocio que implican mantener los datos en infraestructuras controladas. Para desarrollar esta investigación se usó TOGAF como marco de trabajo de arquitectura. Adicionalmente, se incorporaron técnicas modernas como Design Thinking o herramientas de entornos ágiles para poder abrir camino hacia la integración con otros entornos. El resultado es un método de arquitectura que cubre, principalmente, las brechas de seguridad y resiliencia en el diseño logrando un método abierto a integraciones adicionales y cuyos artefactos pueden ser reutilizados por arquitectos de soluciones empresariales.
\end{abstract}

Palabras clave: Computación en nube; nube pública; nube híbrida; arquitectura de nube

\begin{abstract}
This article aims to propose an architecture method that incorporates technological elements commonly used in the implementation of hybrid Cloud and that allows closing the gap in design aspects in solutions for companies that work in industries with regulations. Currently, the use of the public Cloud is the service with the highest growth in the market, guiding the use of on-demand services, known as XasS (All as a Service). In this scenario where data is migrating to public clouds, users are increasingly
\end{abstract}

(C) Los autores. Este artículo es publicado por la Revista Campus de la Facultad de Ingeniería y Arquitectura de la Universidad de San Martín de Porres. Este artículo se distribuye en los términos de la Licencia Creative Commons Atribución No-comercial - Compartir-Igual 4.0 Internacional (https://creativecommons.org/licenses/ CC-BY), que permite el uso no comercial, distribución y reproducción en cualquier medio siempre que la obra original sea debidamente citada. Para uso comercial contactar a: revistacampus@usmp.pe.

https: 
concerned about making safe use of the technology available for the Cloud, complying with their own business regulations that imply keeping the data in controlled infrastructures. To develop this research, TOGAF was used as an architecture framework. Additionally, Design Thinking and other agile tools were incorporated as modern techniques to integrate with different environments. The result is an architectural method that mainly covers security and resilience gaps in the design, achieving an open architecture method for additional integrations and whose artifacts can be reused by enterprise solution architects.

Key words: Cloud computing; public cloud, hybrid cloud; cloud architecture

\section{Introducción}

Las tecnologías de nube desde su aparición fueron siempre un recurso novedoso y de ampla difusión debido, fundamentalmente, a las ventajas que su uso representa a las empresas en distintos campos, especialmente, en la gestión de recursos de TI.

De acuerdo con lo descrito con Patel y Kansara (2021), la demanda de servicios de Cloud Computing ha sido tan importante durante la última década, que incluso ahora es una tecnología conocida como un quinto utility (además del agua, electricidad, gas y teléfono) ya que principalmente es un servicio a demanda. Cloud computing aparece como un servicio basado en un modelo de pago por uso.

El primer caso de uso de esta tecnología aparece como un servicio de nube privada como muchas veces en entornos gubernamentales o de defensa. Youngkon Lee y Ukhyun (2021), describen esta etapa en su trabajo basado en el Reporte de Modernización de TI en el gobierno Federal cuyo documento es actualizado y publicado por el gobierno federal de los estados Unidos. En este documento se introduce el concepto de Public-Private Partnership (PPP) lo cual significa lo que comercialmente evoluciona a lo que se conoce como nube híbrida.

Doheir, Mohamed et al. (2019) proponen un modelo conceptual para mejorar la atención en servicios de salud $\mathrm{e}$ instituciones de la misma industria. En su trabajo publicado en la revista International Journal of Advanced Science and Technology presentan este modelo que se basa en la interacción de múltiples componentes típicos de nubes híbridas con una capa adicional que también es importante mencionar y que se está presentando como un punto crítico en el diseño de todas las arquitecturas en la actualidad, se trata de la conectividad y la interconectividad entre nubes. Este principio nos lleva a profundizar la investigación en un nuevo segmento de empresas que hacen uso de multicloud, es decir, la distribución de cargas de trabajo en distintos ambientes de nube pertenecientes a diferentes proveedores.

Si bien la temática de multicloud puede ser muy amplia y compleja en varios niveles se debe considerar 
como una de las capacidades de nubes híbridas con mucho potencial. Algunas de las razones son expuestas por Boroufar, A. (2020). Software Delivery in Multi-Cloud Architecture en cuyo trabajo se plantean beneficios de alta disponibilidad, flexibilidad, costo -eficiencia y disminución del riesgo desde el punto de vista de seguridad.

La necesidad de incrementar casos de uso en nubes públicas y la demanda del sector de Big data o de data generada por dispositivos de IoT, sensores, ha impulsado la preocupación de generar servicios para atender esta necesidad. Sun, $\mathrm{G}$ et al. (2018) recopilan información de trabajos sobre este tema ahondando sobre los casos de uso que se pueden plantear y utilizar nubes públicas integrándolas con nubes intermedias o con dispositivos conectados en redes privadas.

De esta forma, la transición de datos hacia la nube y su manejo en ella se hace de forma segura y eficiente. Uno de los casos de uso más interesantes es acerca de los dispositivos que recopilan datos de salud de pacientes en tiempo real y lo almacenan en repositorios intermedios que luego son replicados a la nube a través de diferentes medios para realizar análisis de datos y aplicar otras técnicas de análisis con el fin de determinar tendencias, tratamientos, diagnósticos.

Otro punto de vista interesante acerca de la gestión de plataformas de nubes híbridas se plantea como arte del trabajo de Breiter, G., \& Naik, V. K. (2013). En su artículo publicado en IEEE, describen los retos y sobre todo cuales son los principales inhibidores de los proyectos de migración de cargas a nubes públicas. En virtud de esto, muchas empresas sienten la necesidad de mantener el control de sus activos de TI por lo que el reto se plantea en la necesidad de trabajar marcos de trabajo que incluyan verificaciones en seguridad, disponibilidad y resiliencia que impulsen el desarrollo de proyectos de nube.

Ya en el 2014, Donadio, Pasquale \& Fioccola, Giovanni \& Canonico, Roberto \& Ventre, Giorgio. (2014) en su trabajo titulado Network Security for Hybrid Cloud, describían el reto de la seguridad en las arquitecturas de nube híbridas y debido a eso es que empieza a tener mayor visibilidad la necesidad de integrar aspectos de seguridad en arquitecturas que planteen soluciones donde exista transferencia de datos sensibles a la nube.

Otro aspecto relevante con relación a los proyectos de nube híbridas se plantea en el trabajo de P. Segeč et all (2020) en su trabajo Perspective of hybrid cloud computing in higher education y trata acerca del importante rol de las universidades en como utilizan infraestructuras de TI en entornos onpremises y de cómo algunas de ellas, principalmente en Europa, encuentran en proyectos de nube híbridas un camino viable a obtener beneficios de las nubes públicas en entornos educativos. La industria educativa suele ser un facilitador de tecnologías.

Combinando ambos ambientes de nube, privada y pública es posible inclusive colocar a disposición de las empresas capacidades de nube pública en pago por uso a demanda del negocio. Sok, S., Plewnia, C., Tanachutiwat, S., \& Lichter, H. (2020) en su trabajo Optimization of Compute Costs in Hybrid Clouds with Full Rescheduling, 
presentan una forma novedosa de como calendarizar y atender cargas de trabajo a demanda calendarizándolas y manteniendo las capacidades core de aplicaciones e infraestructura crítica en entornos on-premises. Esta posibilidad nos abre un camino nuevo para facilitar la transición hacia la nube.

\section{Método}

Este trabajo de investigación se basa en una metodología descriptiva de las tecnologías que componen una solución de nube hibrida con énfasis en industrias reguladas o con ciertas restricciones de negocio para poder mover cargas de trabajo a una nube pública.

Para tal fin, se revisan dos casos de uso dondeseaplicaron diferentesherramientas para sustentar una arquitectura basada en las buenas prácticas donde se aplican métodos de arquitectura basados en TOGAF como método principal o marco de trabajo sobre el cual referenciamos los artefactos y herramientas usados en la investigación.

Como resultado se presenta un método de arquitectura de cinco etapas en las que se realizan definiciones de diseño de una solución para nubes híbridas en entornos regulados. Debido a que los entornos regulados pueden ser una fuente amplia de casos de uso, plantearemos el ámbito de dos industrias: Industria de seguros y la Industria de cuidado de la salud.

Debido a las restricciones de uso en cuanto a presupuesto y complejidad que se platean al inicio de este proyecto, establecemos un método que se compone cinco pasos bien definidos y que se pueden adaptar a los proyectos que se describen en esta investigación. Cada uno de los pasos se compone de artefactos o entregables reutilizables durante el ciclo de vida de un proyecto de TI. Adicionalmente se irán utilizando herramientas que aportarán al desarrollo de los entregables dándole a los proyectos mayor soporte durante el diseño y la implementación.

\section{Visión}

De manera similar a lo propuesto en el framework de TOGAF, en esta etapa se propone una etapa en la que se pueda establecer la Visión del proyecto orientándolo al logro de objetivos de negocio o metas organizacionales.

Para esto se necesitan identificar los stakeholders correctamente e interactuar con ellos de forma constante para identificar las posibles brechas que tengamos que cubrir más adelante.

En la etapa 1, se deberán validar y confirmar que los requerimientos de negocio se encuentren alineados a la expectativa del usuario o cliente. De esta manera, será más fácil definir un alcance del servicio que cubra la demanda del Cliente.

Siguiendo lo indicado, se deberán documentar tres entregables:

- Requerimiento clave de negocio

- Alcance de servicio

- Requerimientos funcionales y no funcionales

Para lograr el objetivo de documentar correctamente esta etapa se pueden utilizar herramientas de uso tradicional en la formulación de proyectos. 


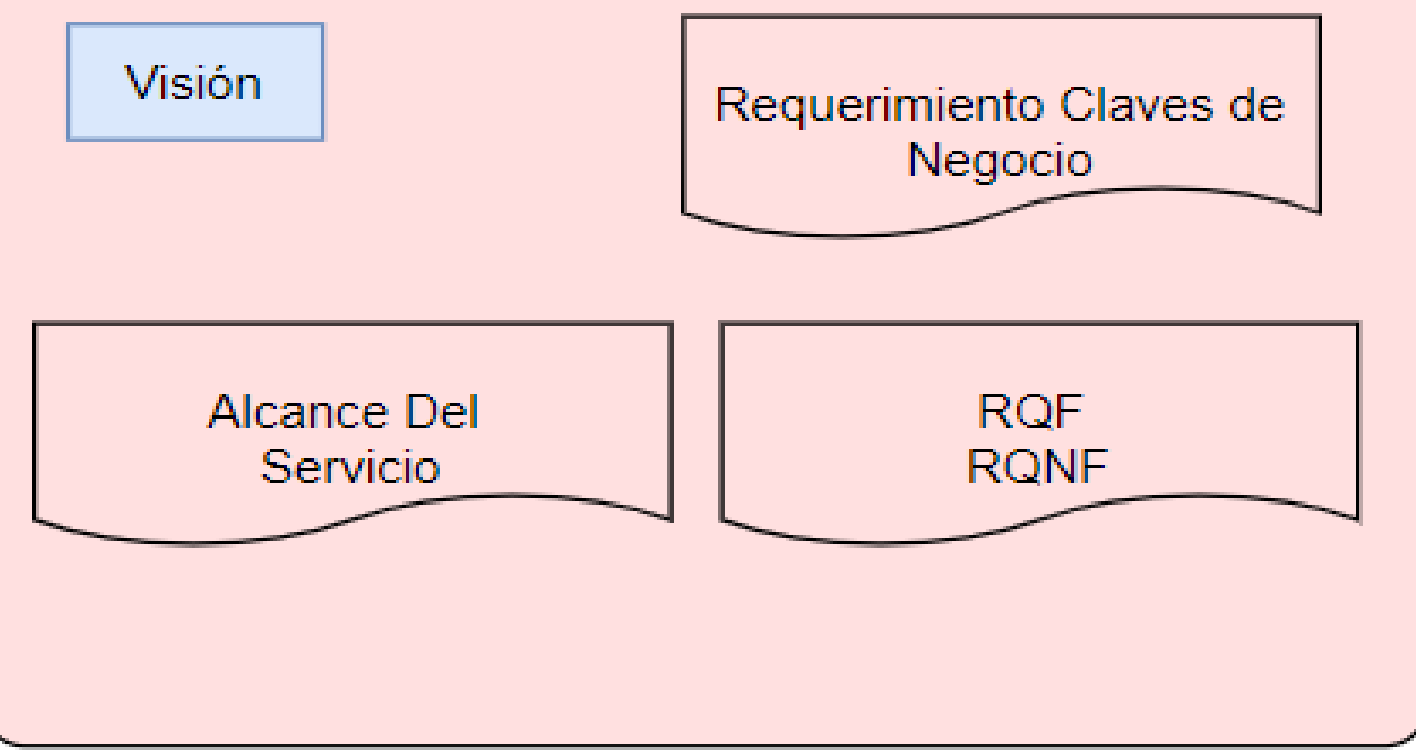

Figura 1. Etapa 1 del método propuesto: visión

\section{Co-creación}

Para completar la etapa 2 del método exitosamente, es necesario establecer un canal de comunicación eficiente con los stakeholders y teniendo claro lo desarrollado en la primera etapa, estaremos listos para trabajar con el cliente en una etapa e co-creación donde el objetivo es establecer las bases del diseño arquitectónico para la solución.

Durante este proceso es importante seguir alimentando y validando el alcance del servicio de tal manera que, si hay cambios, estos sean documentados.

Como entregables definidos en esta etapa tenemos:

- Decisiones de arquitectura

- Documentación de matriz RACI
(Responsible, Accountable, Consulted, Informed)

- Documentación de matriz RAID (Risks, Assumptions, Issues, Dependencies)

En esta etapa, se puede usar el marco de trabajo Design Thinking como parte del proceso de co-creación con el Cliente o los stakeholders para tener no solo el alcance bien estructurado sino también validar los objetivos de la solución.

En esta fase, son primordiales las herramientas de colaboración que podamos usar de forma efectiva sin necesidad de incrementar la complejidad del proceso. Como herramientas sugeridas podemos mencionar Trello, Miro, Storytelling, demostraciones o workshops. 


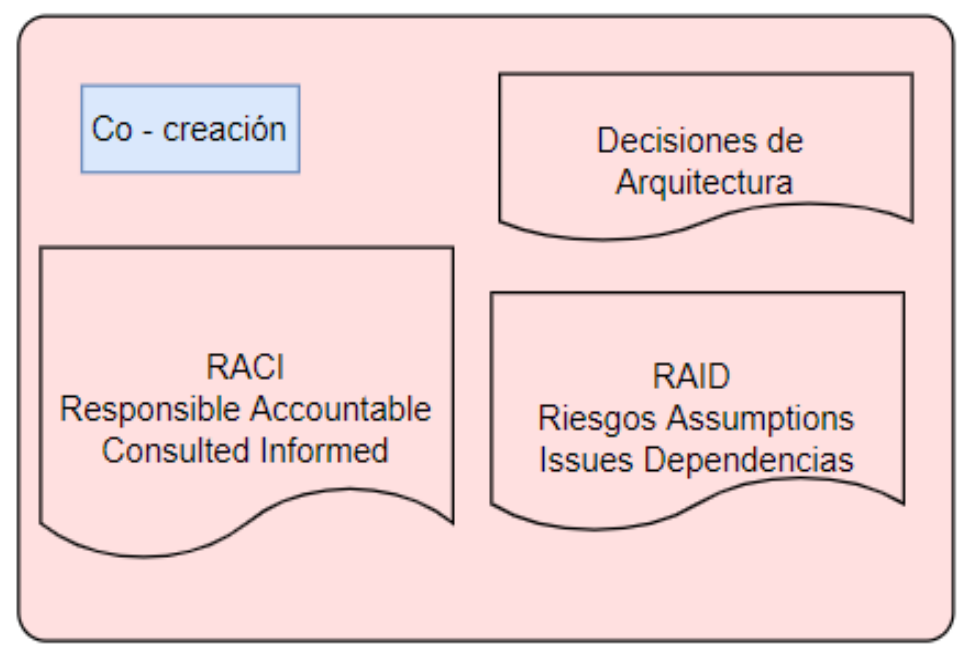

Figura 2. Etapa 2 del método propuesto: co-creación

\section{Diseño}

En la etapa 3, de Diseño se tendrán que poner en práctica las habilidades de arquitectura no solo a nivel técnico sino también a nivel de conocimiento del negocio y de los procesos clave que se verán involucrados en el desarrollo de la solución. Por esa razón, es importante mantener el vínculo creado durante las primeras etapas de interacción con los stakeholders.

Los entregables que son parte de esta
- Arquitectura de alto nivel

- Arquitectura de aplicaciones

- Arquitectura de infraestructura

Las herramientas disponibles son múltiples. Sin embargo, siguiendo el framework elegido, es recomendable utilizar una herramienta de modelamiento que incluya objetos de Archimate ya que es probable que tengamos que usar elementos de arquitectura empresarial en proyectos que requieren mayor nivel de detalle de las relaciones de negocio. etapa son:

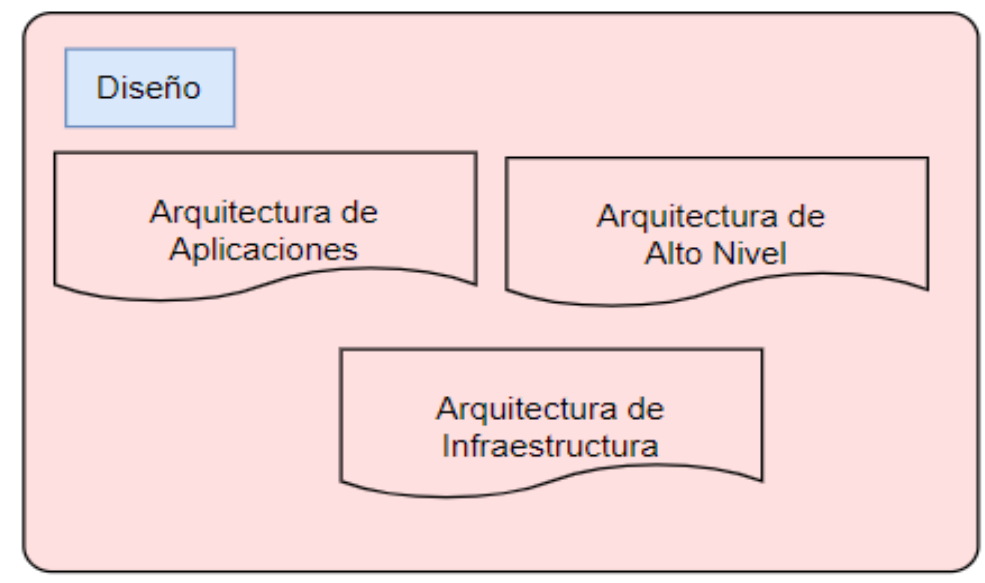

Figura 3. Etapa 3 del método propuesto: diseño

\section{Seguridad}

Si bien durante todo el ciclo de vida del proyecto se deben revisar los aspectos relacionados con la seguridad en general, es importante tomarse un tiempo para considerar aspectos de seguridad de la solución que estamos diseñando. 
En la etapa 4, del método debemos enfocarnos en documentar y plantear la gestión de riesgos, definir la seguridad de las interfaces, validar los elementos de seguridad perimetral y resiliencia.

Sobre el tema de resiliencia, es muy importante verificar con los stakeholders que sus expectativas de redundancia y alta disponibilidad del servicio son viables y que pueden implementarse a nivel técnico y funcional

Adicionalmente, es necesario ayudar al cliente a definir procedimientos de disaster recovery si es que no los tuvieran ya definidos $y$, sobre todo, considerando que este método se enfoca en industrias reguladas, es importante documentar que todas las regulaciones exigidas por la industria o sector en el que se desenvuelven estas empresas sean cumplidos por la infraestructura que se está diseñando y que en el futuro va a soportar las funcionalidades del negocio.

Para esta etapa se plantean los siguientes entregables:
- Gestión de riesgos

- Definición de interfaces

- Validación de elementos de seguridad y resiliencia

- Validación de regulaciones

Algunas herramientas útiles en esta etapa son la documentación del proceso de gestión de riesgos. Esto se puede realizar a través de un documento que reúna todas las condiciones y alcance de seguridad para la solución. Por ejemplo, políticas de compliance, frecuencias de parchados, procedimientos de Health Checking periódicos para asegurar que las políticas de seguridad se sigan cumpliendo.

En esta etapa es muy importante el involucramiento del área de seguridad del cliente ya que se trata de cubrir todos los puntos críticos del servicio y sobre todo en común acuerdo con los stakeholders.

Debido a que este método aspira cubrir las necesidades de empresas que requieren entornos de nube híbridos, se requiere conocer los detalles de conectividad de los ambientes requeridos para la solución.

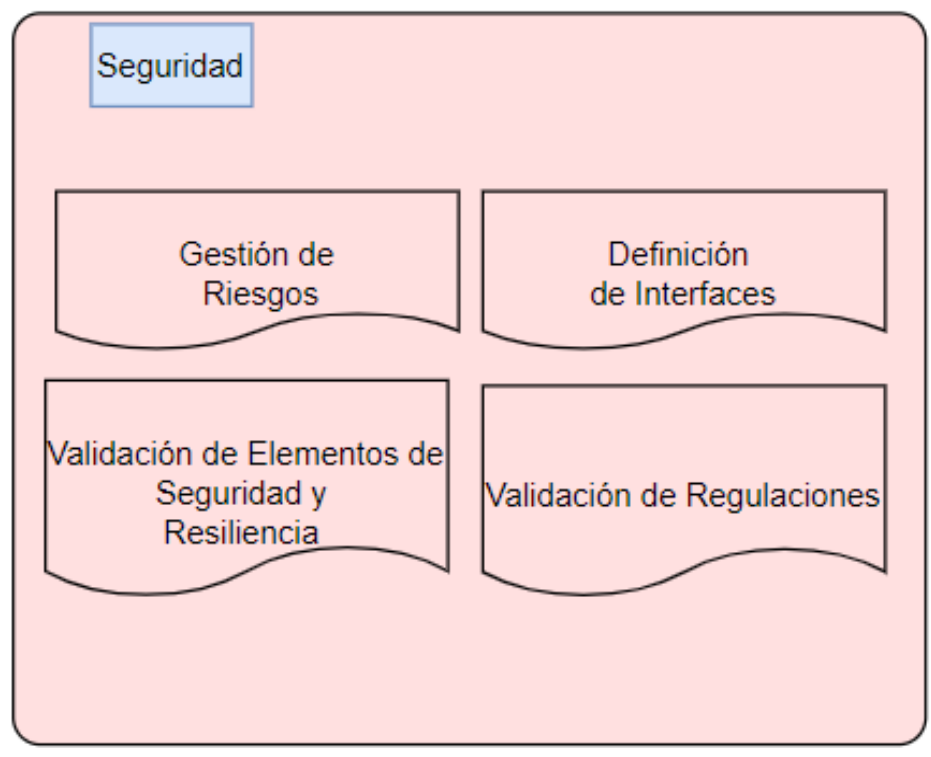

Figura 4. Etapa 4 del método propuesto: seguridad 


\section{Mejora continua}

Una vez que se han cubierto las etapas anteriores del método propuesto, se deben establecer procedimientos claros para la gestión de requerimientos y cambios, así como el feedback constante de los stakeholders. En la etapa 5, es importante verificar que se haya cumplido el plan de pruebas, así como los objetivos de las migraciones o despliegues que se identificaron durante la etapa de diseño.

En esta etapa, el rol de gobierno de la arquitectura y del proyecto se convierten en roles críticos ya que es importante recibir los requerimientos del cliente a través de una herramienta de control y seguimiento de los requerimientos y de los cambios ejecutados.

En este punto del ciclo de vida de la solución, el cliente debería tener claro un flujo claro y detallado de como introducir un requerimiento o una solicitud de cambio. Esto puede derivar en una evaluación del impacto y realizar la ejecución de forma controlada.

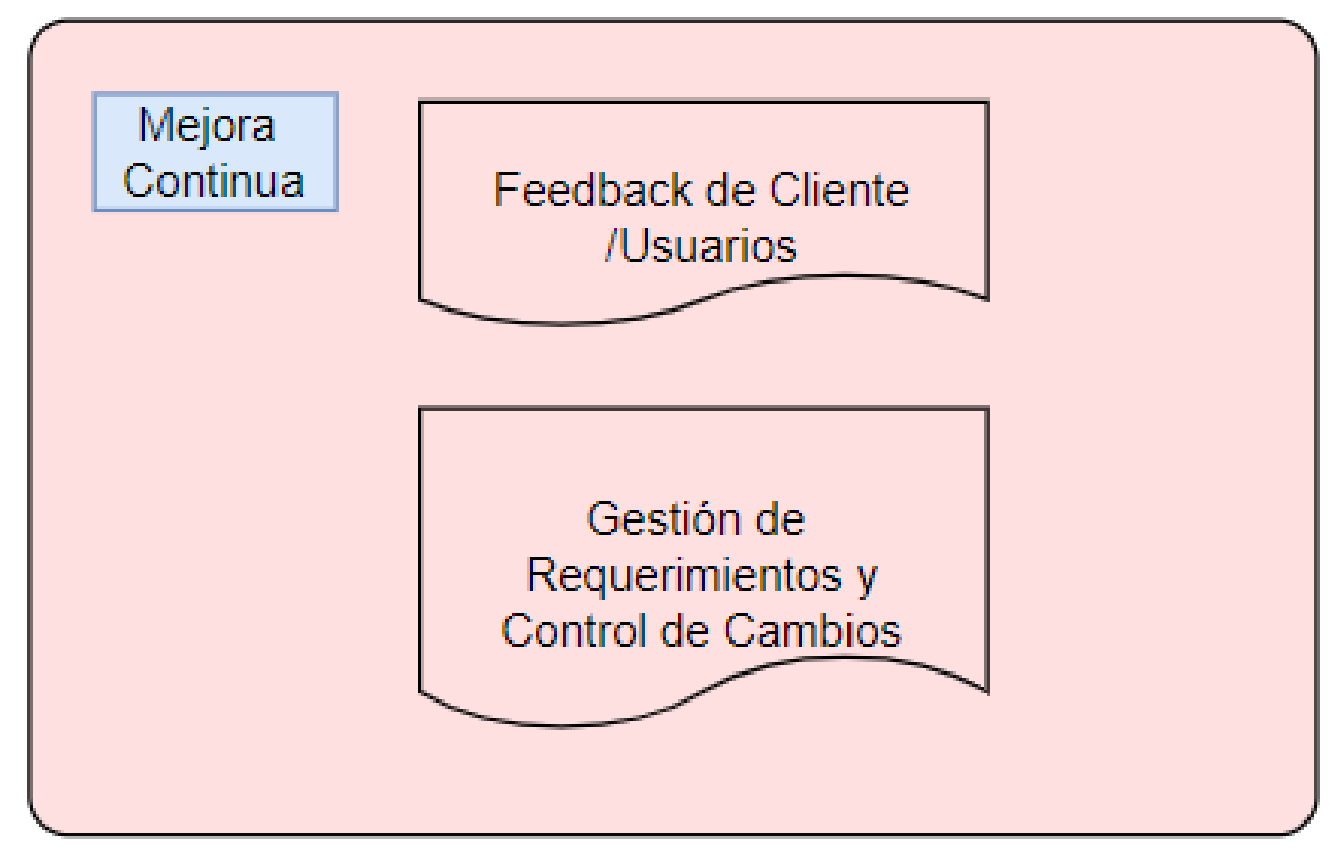

Figura 5. Etapa 5 del método propuesto: Mejora continua

\section{Integración con metodologías ágiles}

Las metodologías ágiles se han convertido en parte clave de los procesos internos y externos de las empresas, debido a las ventajas que están disponibles al incorporarlas en la operación y gestión de proyectos.
En el alcance del presente trabajo se considera que durante el ciclo de vida del proyecto se integren roles y procesos adicionales en el diseño de una solución bajo este marco de trabajo. 


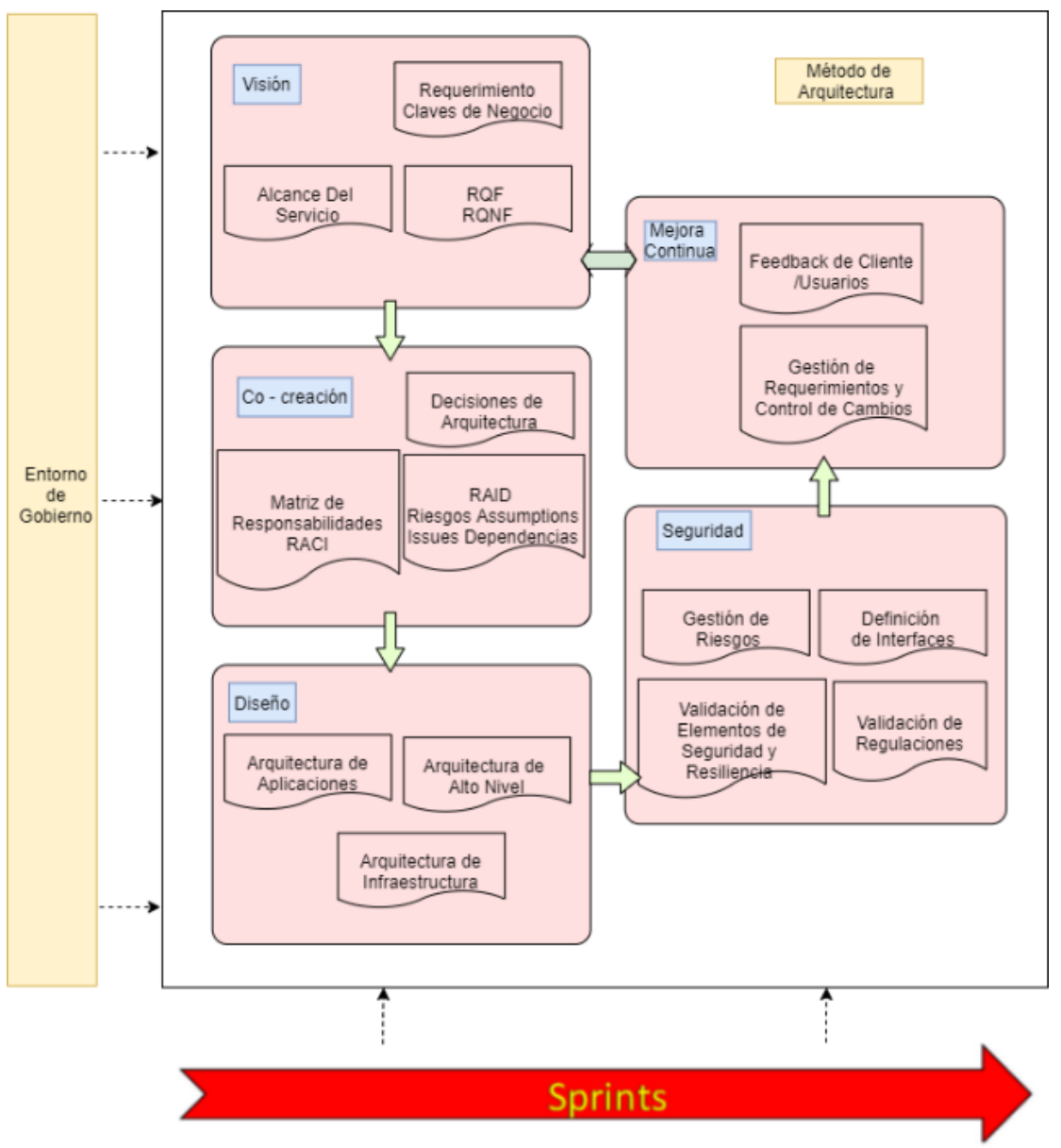

Figura 6. Integración del método de arquitectura con metodologías ágiles y un entorno de gobierno

\section{Entorno de gobierno}

Los entornos de gobierno en proyectos de arquitectura suelen ser clave durante el diseńo y el ciclo de vida de estos. Permite que las soluciones se mantengan dentro del alcance definido en las primeras etapas y se cumpla además con los requerimientos de compliance y entornos regulados.

Adicionalmente, es importante contar con una estructura de gobierno por que los proyectos de arquitectura necesitan de principios y políticas asociadas a guías de implementación. Es por eso por lo que una estructura de gobierno que cubra todas las fases del método facilita el logro de los objetivos, así como proporciona los estándares preventivos y correctivos cuando se encuentra algún desvío del proyecto en cualquiera de las fases.

El entorno de gobierno debe ser un proceso colaborativo con los demás componentes de los proyectos como el diseño mismo a nivel de arquitectura, el desarrollo de los componentes, operación infraestructura, reglas de negocio y gestión del proyecto mismo. Esto sucede en cada iteración.

\section{Impacto de metodologías ágiles en el método de arquitectura}

Se pueden usar diferentes herramientas y técnicas durante la ejecución del método de arquitectura propuesto. Es 
clave además que se ejecute un proceso iterativo usando sprints con pequeñas iteraciones para obtener validación a través del feedback de los stakeholders y las áreas de implementación quienes ya suelen trabajar en entornos ágiles.

El dividir el trabajo en sprints facilitará una rápida adaptación del proyecto y además gestionar los cambios adecuadamente. Esto genera valor a las soluciones ya que es posible adaptar los cambios necesarios sin rehacer el proyecto.

En todos los casos será necesario establecer un punto de inicio para alinear el requerimiento y expectativas del cliente con las capacidades del equipo que implementará la solución. En este punto es recomendable que el equipo de arquitectura lidere las reuniones y documente las iteraciones.

\section{Resultados y discusión}

Se aplicó el método de arquitectura propuesto en dos casos de uso que se encuentran en el rango de las industrias de entornos regulados. Empresa del sector de seguros y Empresa del sector cuidado de la salud. Para fines de alcance del artículo se presentan las arquitecturas de ambos casos de uso y se dispondrá de la documentación en un repositorio interno.

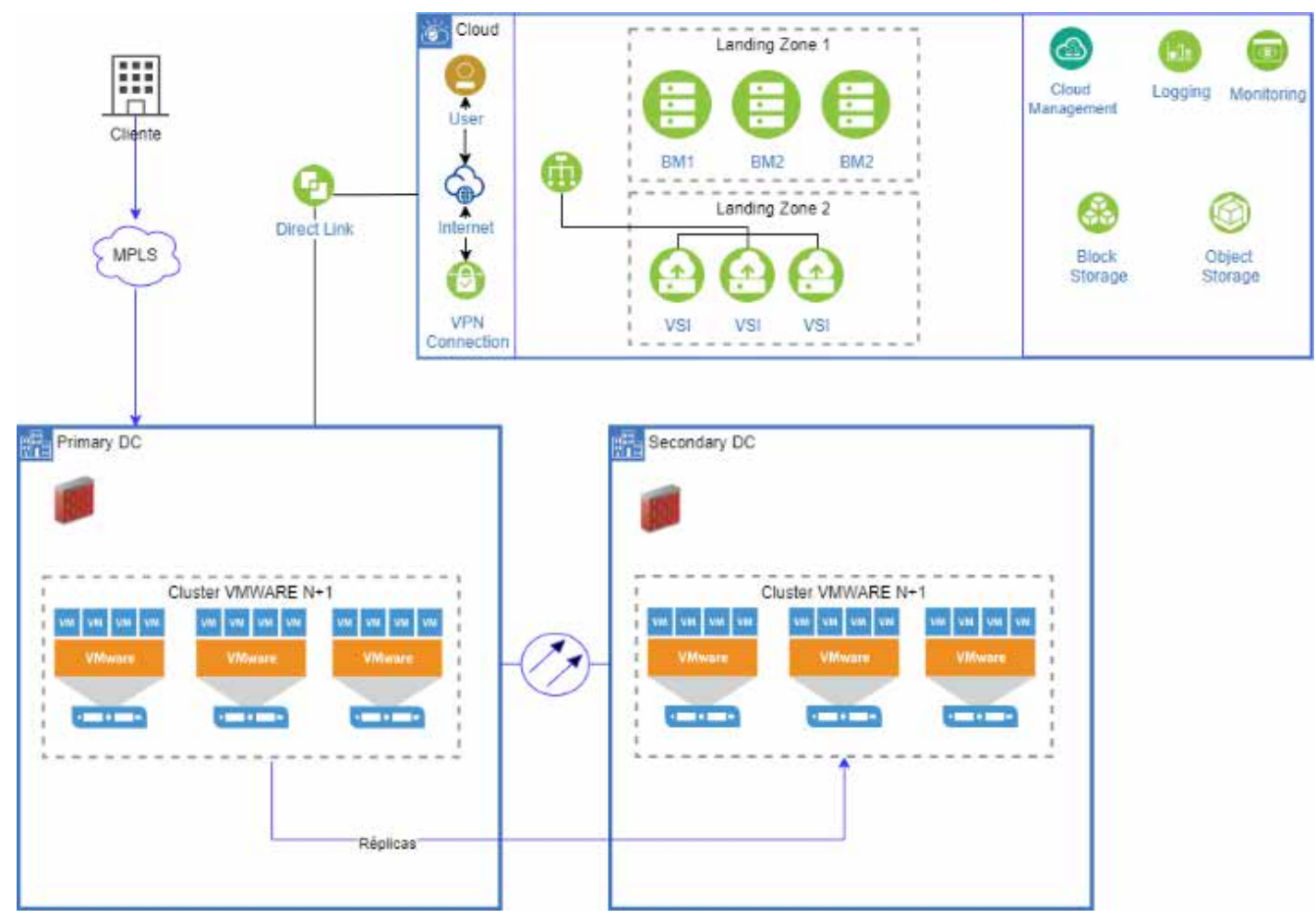

Figura 7. Diagrama de arquitectura para empresa de industria de seguro

En el caso de uso de empresa de sector seguros se tuvieron en consideración los cinco pasos propuestos por el método de arquitectura, considerando que la necesidad urgente era la de distribuir las cargas de trabajo en dos sites on-premises y mantener un entorno de nube pública con los requerimientos de seguridad y certificaciones de industria regulada. 


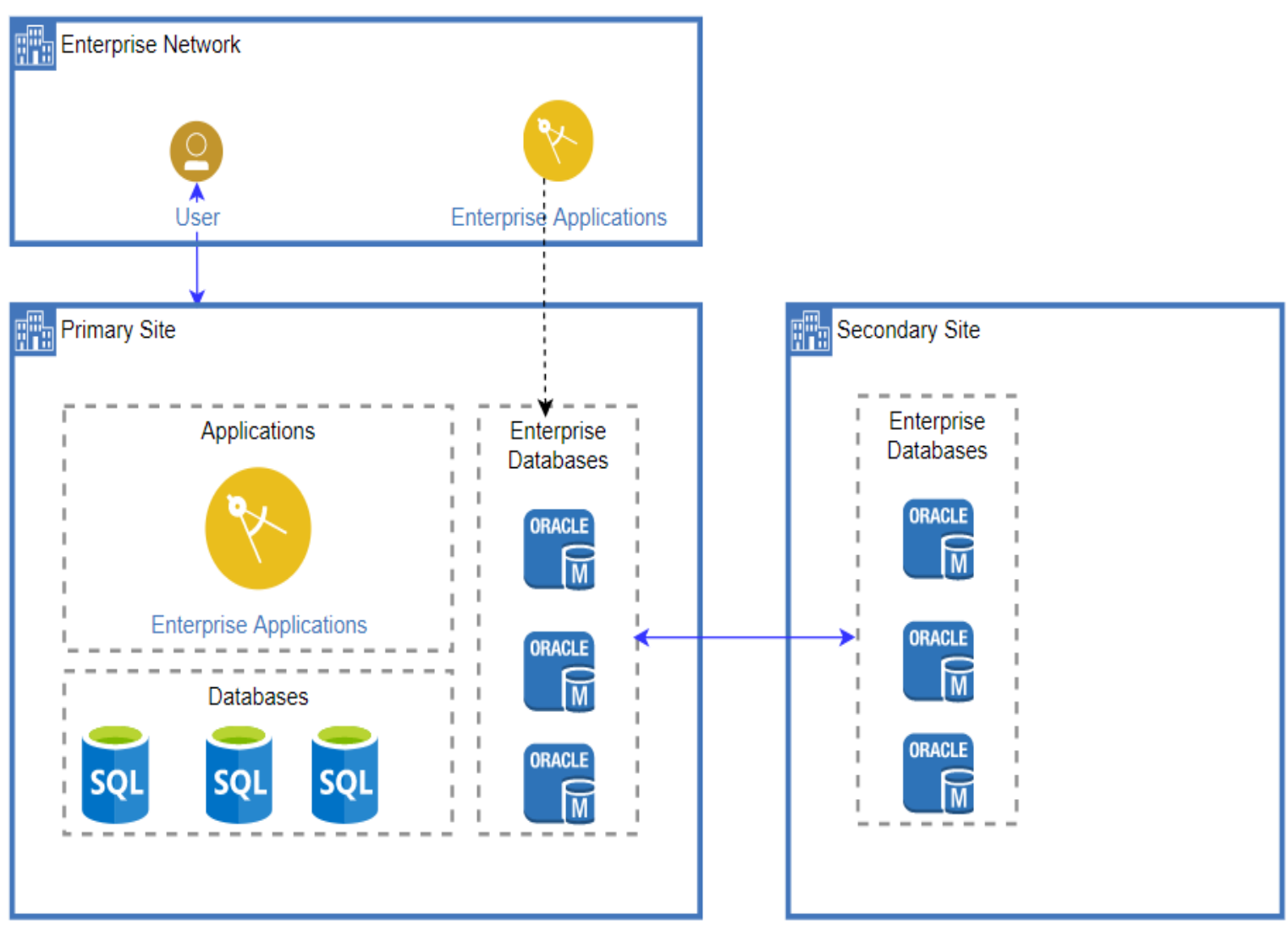

Figura 8. Diagrama de aplicaciones para empresa de sector seguros

\section{Caso de uso: Empresa del sector Salud}

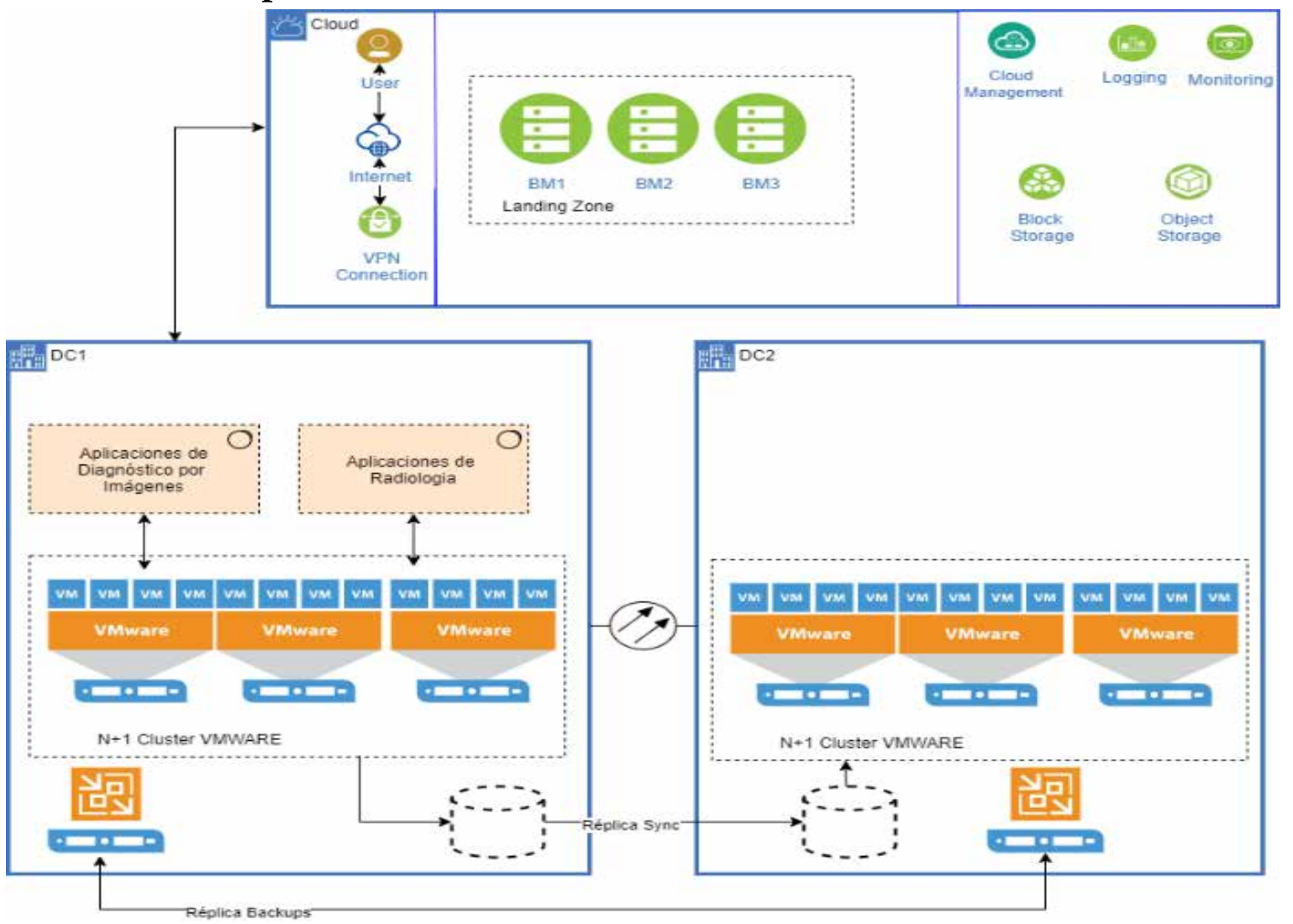

Figura 9. Diagrama de arquitectura de aplicaciones de empresa sector salud 
En el caso de uso de empresa del sector salud, se presenta un escenario en el que la definición de arquitectura y los componentes elegidos están lineados con el objetivo de negocio de la empresa que se ve adelantado por la pandemia global COVID-19.
La necesidad de poder contar con información de ayuda a diagnóstico a través de imágenes es primordial y se pone en práctica en el proyecto mencionado.

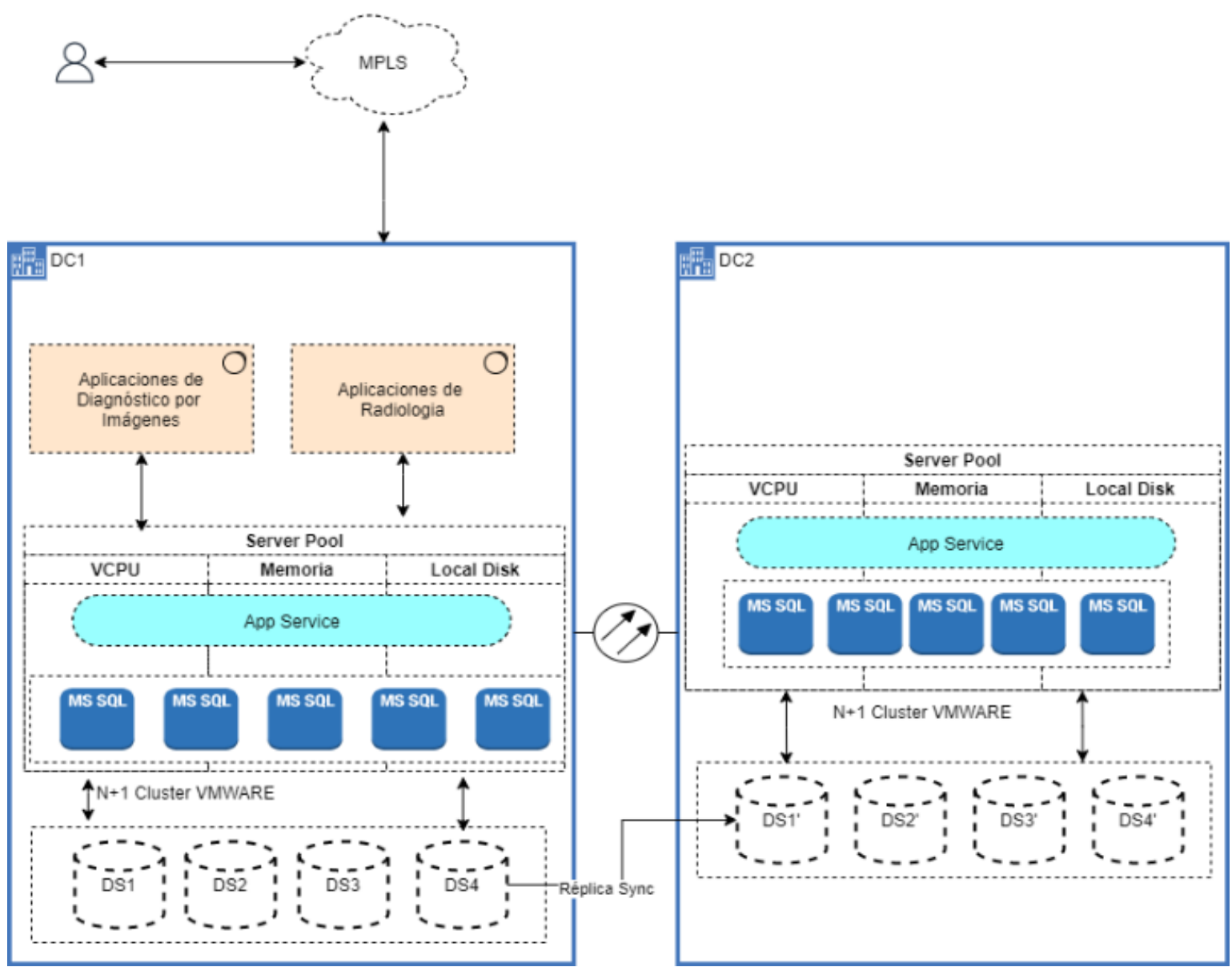

Figura 10. Diagrama de aplicaciones para empresa del sector salud

En el trabajo de investigación desarrollado se presenta un método de arquitectura para empresas cuya solución incluye entornos de trabajo en nubes híbridas y se muestra como resultado la arquitectura detallada de las soluciones que fueron aplicadas en proyectos reales donde el principal objetivo era disponer de recursos de nube publica manteniendo servicios de infraestructura en entornos de nubes privadas o de tipo on-premise.
Para evaluar los resultados se utilizaron técnicas de medición de satisfacción del cliente basándose en su experiencia, es decir no solo la percepción del equipo técnico o el arquitecto que diseñó las soluciones sino también evaluando el nivel de satisfacción del cliente a través de la experiencia de servicio.

Para esto se usó la técnica de NPS (Net Promoter Score) que busca a través de una pregunta especifica determinar el nivel de 
compromiso del cliente o usuario con la solución. La pregunta busca direccionar la respuesta del cliente en base a que tan cómodo o satisfecho se siente con relación a recomendar el servicio o la experiencia a un amigo o colega en la escala de 0 a 10 . Basándose en las respuestas, se clasifica a los clientes en tres categorías: Detractores, Pasivos y Promotores

Para este trabajo de investigación se están presentando dos casos de uso que recibieron puntaje de Promoter por los evaluadores. Sin embargo, hay consideraciones que deben tomarse en cuenta como parte de las lecciones aprendidas para el trabajo a futuro.

La arquitectura de TI es una disciplina técnica que permite diseñar y crear de forma metodológica una solución que va a satisfacer una necesidad del cliente o del negocio. Para lograr este desafío encontramos múltiples herramientas y técnicas. Algunas de ellas se han revisado en este trabajo de investigación y han servido de base para presentar el método de arquitectura que ha servido de guía en la creación de la solución propuesta.

El escenario de pandemia, que estamos viviendo en la actualidad, ha servido para impulsar drásticamente la utilización de recursos y tecnologías que hasta hace poco se discutían si era o no factible usarlas. Es por eso por lo que contar con la mayor documentación existente acerca de los procesos involucrados para diseñar arquitecturas costo - eficientes y viables a nivel técnico es un punto crítico de éxito en proyectos que incorporan nubes híbridas en las soluciones propuestas.

La utilización de nube híbrida es el camino más viable para acceder a los beneficios de utilización de nube considerando todos los aspectos de seguridad, resiliencia y regulaciones existentes. En este contexto, las empresas con regulaciones de industria son ideales para poner en práctica el método propuesto considerando que logramos cubrir los puntos críticos en el desarrollo de la arquitectura de soluciones.

\section{Conclusiones}

El camino de desarrollo de la nube híbrida está bastante marcado por el crecimiento del mercado de las nubes públicas. Esto es un indicador que, en el futuro a corto y mediano plazo, las empresas buscarán optimizar sus recursos de TI con una mezcla de soluciones que incorporen necesidades de entornos onpremise.

El uso de métodos de arquitectura durante el diseño de una solución favorece el éxito de los proyectos de TI en general y específicamente para el caso de proyectos donde se incluye infraestructura híbrida es crucial para determinar los posibles puntos de falla y asegurar el nivel de disponibilidad y resiliencia.

La utilización de entornos de nube basada en gestión multicloud habilita a las empresas a tener mayor espectro de servicios disponibles. Sin embargo, esto aumenta el nivel de complejidad y detalle en aspectos relacionados con seguridad, disponibilidad y conectividad.

El gobierno de TI es clave en proyectos de nube hibrida ya que es necesario introducir las variables de calidad del servicio, compliance y evaluación de regulaciones de la industria en la cual se desempeñan las empresas. 
Incorporar procesos y técnicas ágiles fortalece el gobierno de TI y la eficiencia operativa del diseño de arquitectura de esta clase de proyectos. Se puede tomar lo mejor de la gestión de proyectos ágiles considerando las iteraciones durante el ciclo de vida del proyecto y hacer que las mejoras y cambios sean ejecutados con éxito

\section{Referencias}

Boroufar, A. (2020). Software Delivery in Multi-Cloud Architecture [Laurea, Politecnico di Torino]. https:// webthesis.biblio.polito.it/16753/

Breiter, G., \& Naik, V. K. (2013). A Framework for Controlling and Managing Hybrid Cloud Service Integration. 2013 IEEE International Conference on Cloud Engineering (IC2E), 217224. https://doi.org/10.1109/ IC2E.2013.48

Doheir, M., Basari,A.H., Elzamly,A., Hussin, B., Yaacob, N. M., \& Al-Shami, S. S. A. (2019). The New Conceptual Cloud Computing Modelling for Improving Healthcare Management in Health Organizations. International Journal of Advanced Science and Technology, 28(1), 351-362.

Donadio, P., Fioccola, G. B., Canonico, R., \& Ventre, G. (2014). Network security for Hybrid Cloud. 2014 Euro Med Telco Conference (EMTC), 1-6. https://doi.org/10.1109/ EMTC.2014.6996640

Lee, Y., \& Lee, U. (2021). Reference Architecture and Operation Model for PPP (Public-PrivatePartnership) Cloud. Journal of Information Processing Systems, 17(2), 284-296.

Liu, A., \& Yu, T. (2018). Overview of Cloud Storage And Architecture
(SSRN Scholarly Paper ID 3649074). Social Science Research Network. https://papers.ssrn.com/ abstract $=3649074$

Patel, H. B., \& Kansara, N. (2021). Cloud Computing Deployment Models: A Comparative Study (SSRN Scholarly Paper ID 3832832). Social Science Research Network. https://papers. ssrn.com/abstract $=3832832$

Segeč, P., Moravčik, M., Kontšek, M., \& Papán, J. (2020). Perspective of hybrid cloud computing in higher education. 2020 18th International Conference on Emerging eLearning Technologies and Applications (ICETA), 586592. https://doi.org/10.1109/ ICETA51985.2020.9379182

Sok, S., Plewnia, C., Tanachutiwat, S., \& Lichter, H. (2020). Optimization of Compute Costs in Hybrid Clouds with Full Rescheduling. 2020 IEEE International Conference on Smart Cloud (SmartCloud), 35-40. https://doi.org/10.1109/ SmartCloud49737.2020.00016

Sun, G., Guan, S., Ramachandran, M., Li, J., \& Liao, D. (2018). Big Data and Internet of ThingsFusion for different services and its impacts-Teesside University's Research Portal. https://research. tees.ac.uk/en/publications/bigdata-and-internet-of-thingsfusionfor-different-services-and- 